\title{
Priapismo de alto flujo postraumático en paciente pediátrico tratado con embolización arterial selectiva
}

\author{
Postraumatic high-flow priapism in pediatric patient treated with selective \\ arterial embolization
}

\author{
Dr. Roberto Molina Escudero ${ }^{a}$ Dr. José Hernández Atance ${ }^{b}$ Dra. María del Mar Ballesteros García $y$ \\ Dr. Álvaro Páez Borda ${ }^{a}$
}

\begin{abstract}
RESUMEN
El priapismo arterial es una rara patología en pacientes pediátricos, originada por una fístula entre la arteria cavernosa y los sinusoides del cuerpo cavernoso, habitualmente secundaria a un traumatismo perineal.

Presentamos el caso de un varón de 16 años con priapismo arterial de 5 días de evolución tras una caída a horcajadas, tratado satisfactoriamente mediante embolización arterial supraselectiva con material reabsorbible. Seis meses después, el paciente presenta detumescencia completa sin disfunción eréctil asociada.

La embolización arterial en pacientes pediátricos es compleja debido al menor calibre arterial y la necesidad de control de la arteria pudenda interna contralateral para evitar el desarrollo de complicaciones. La utilización de material reabsorbible permite disminuir el riesgo de disfunción eréctil posterior y, aunque presenta un mayor índice de recurrencias, constituye una alternativa eficaz en el tratamiento de esta patología.

Palabras clave: priapismo arterial, embolización terapeútica, disfunción eréctil.
\end{abstract}

\section{ABSTRACT}

Arterial priapism is a rare condition in pediatric patients, caused by a fistula between the cavernous artery and the sinusoids of the corpus cavernosum, usually secondary to perineal trauma. We report the case of a 16 year old child with arterial priapism of 5 days duration following a fall astride, successfully treated by superselective arterial embolization with absorbable material. Sixmonths later the patient had complete detumescence without secondary erectile dysfunction.

Arterial embolization in pediatric patients is complex due to the smaller arterial size and the need to control the contralateral internal pudendal artery to prevent the development of complications. The use of absorbable material helps reduce the risk of subsequent erectile dysfunction and, although it has
\end{abstract}

a. Servicio de Urología.

b. Servicio de Radiología Vascular Intervencionista.

c. Servicio de Pediatría.

Hospital Universitario de Fuenlabrada. Madrid.

Correspondencia:

Dr. Roberto Molina Escudero: robersescu@hotmail.com

Financiamiento: Ninguno.

Conflicto de intereses: Ninguno que declarar.

Recibido: 10-11-2014

Aceptado: 19-2-2015 a higher rate of recurrence, is an alternative for the treatment of this pathology.

Key words: arterial priapism, embolization, therapeutic, erectile dysfunction.

http:/ /dx.doi.org/10.5546/aap.2015.e199

\section{INTRODUCCIÓN}

El priapismo es una erección mantenida más de 4 horas en ausencia de estímulo sexual. Existen dos tipos fundamentales: isquémico o de bajo flujo, y arterial o de alto flujo. ${ }^{1}$ El primero es la forma infantil más frecuente y se asocia a la anemia de células falciformes en el $60 \%$ de los casos. Consiste en la imposibilidad del retorno sanguíneo desde los cuerpos cavernosos como consecuencia de la obstrucción al flujo venoso, lo que provoca un síndrome compartimental con erección dolorosa y constituye una emergencia urológica por el riesgo de isquemia, necrosis y fibrosis del tejido eréctil. ${ }^{2}$ El priapismo arterial se produce por el desarrollo de una fístula entre una arteria cavernosa y los sinusoides del cuerpo cavernoso, habitualmente originada por un traumatismo pélvico o perineal, que condiciona un hiperflujo sanguíneo continuo al cuerpo cavernoso y provoca una erección no dolorosa e incompleta $\sin$ isquemia asociada. ${ }^{3}$

\section{CASO CLÍNICO}

Presentamos el caso de un varón de 16 años sin antecedentes médicos quirúrgicos de interés, que acude a Urgencias por presentar una erección mantenida de 5 días de evolución, 12 horas después de una caída a horcajadas sobre una valla. La exploración física evidenció un hematoma perineo escrotal sin lesión de los genitales externos. El pene mostraba una erección completa, no dolorosa, de rigidez parcial, con participación de todo el cuerpo esponjoso, que incluía el glande. Se realizó una punción del cuerpo cavernoso utilizando una aguja de 19 $\mathrm{G}$ y se obtuvo sangre de aspecto arterial. La gasometría realizada evidenció un pH de 7,40; 
$\mathrm{PO}_{2}$ de $80 \mathrm{mmHg}$ y $\mathrm{PCO}_{2}$ de $40 \mathrm{mmHg}$, compatible con el diagnóstico de priapismo de alto flujo.

Inicialmente, el paciente fue manejado de forma conservadora mediante compresión perineal y aplicación de hielo local intermitente durante 48 horas, sin mejoría. Ante la persistencia de la erección, bajo sedación, se realizó una arteriografía por vía femoral derecha, que evidenció una fístula entre la arteria cavernosa y el cuerpo cavernoso izquierdos (Figura 1), que fue embolizada mediante el uso de material reabsorbible (Figura 2). El posoperatorio transcurrió sin incidencias y el paciente fue dado de alta 24 horas después con detumescencia completa. Seis meses después, el paciente mantiene la detumescencia y presenta erecciones nocturnas espontáneas. La ecografía doppler demuestra presencia de flujo arterial en los cuerpos cavernosos.

\section{DISCUSIÓN}

El objetivo inicial en los pacientes con priapismo es discernir el tipo que presenta el paciente para distinguir los casos que constituyen una emergencia (isquémico) de los que se pueden tratar de forma diferida (alto flujo). Inicialmente, la historia clínica y la exploración física pueden orientar hacia el tipo de priapismo. La presencia de una erección dolorosa y completa orienta hacia priapismo de bajo flujo, mientras que el ante-

Figura 1. Arteriografía que demuestra fuga de medio de contraste desde la arteria cavernosa izquierda al cuerpo cavernoso ipsilateral (flecha)

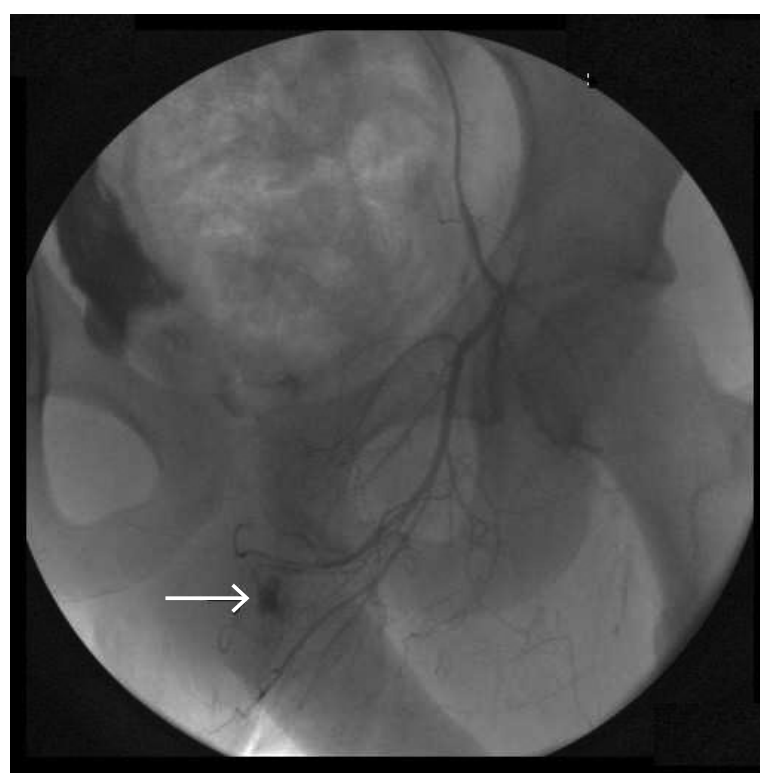

cedente de traumatismo perineal orientará hacia priapismo arterial. En todos los casos, es necesaria la confirmación mediante gasometría del cuerpo cavernoso, que permite realizar el diagnóstico definitivo de priapismo arterial o isquémico según los valores de $\mathrm{pH}, \mathrm{PCO}_{2}$ y $\mathrm{PCO}_{2}$ (Figura 3). ${ }^{4}$

El priapismo arterial es una manifestación poco común de la enfermedad; hasta la fecha, se han encontrado solo 53 casos publicados en pacientes pediátricos. ${ }^{5}$ La erección suele aparecer hasta 7 días después del traumatismo, debido a que las erecciones nocturnas provocan la ruptura del coágulo formado tras este, lo que permite el desarrollo de una fístula entre la arteria cavernosa y los sinusoides. Una vez desarrollada, se produce un incremento del flujo sanguíneo que entra en los cuerpos cavernosos y estimula la enzima óxido nítrico sintasa, la cual incrementa la producción de óxido nítrico y GMPc, que provocan la relajación del músculo liso de los cuerpos cavernosos y perpetúan la erección. ${ }^{6}$

Debido a que el mecanismo fisiopatológico consiste en un hiperflujo sanguíneo, el priapismo arterial no constituye una emergencia urológica, puesto que no implica un daño isquémico en los cuerpos cavernosos. Por este motivo, existen trabajos que abogan por un manejo conservador frente al abordaje tradicional mediante embolización arterial, aunque no existe consenso sobre el tratamiento de elección en esta patología.?

FiguRA 2. Arteriografía que evidencia la resolución de la fístula mediante embolización selectiva (flecha)

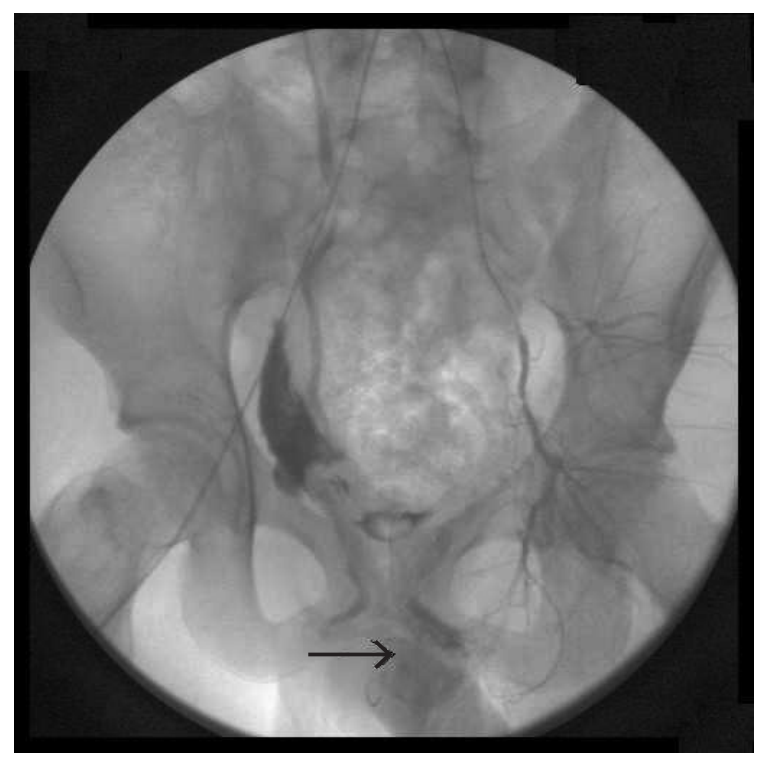


El tratamiento conservador consiste en la observación o compresión perineal guiada o no por ecografía doppler. Se ha descrito en casos clínicos aislados, y se ha logrado la resolución del cuadro en el $62 \%$ de los pacientes, principalmente en las 6 primeras semanas y, sobre todo, en aquellos en los que no puede documentarse la fístula arteriocavernosa mediante ecografía doppler, posiblemente porque esta sea de muy pequeño tamaño. ${ }^{8-10}$ Las razones fundamentales a favor de este abordaje son la ausencia de utilización de medidas invasivas, que en pacientes pediátricos, precisan de anestesia general o sedación, y disminuir el riesgo de complicaciones asociadas a dichos procedimientos, como la disfunción eréctil.

Sin embargo, Tonseth et al. ${ }^{11}$ exponen que la elevada tensión de oxígeno mantenida sobre los cuerpos cavernosos puede provocar fibrosis y disfunción eréctil posterior, por lo que recomiendan realizar un tratamiento activo lo antes posible.

Tradicionalmente, el tratamiento del priapismo de alto flujo en adultos ha consistido en la embolización y oclusión de la fístula arteriocavernosa, lo que permite su cierre. En la población pediátrica, existen muy pocos casos descritos en la literatura, puesto que requiere de radiólogos intervencionistas especializados, debido a que el calibre de las arterias es menor que en los adultos, lo que dificulta la embolización selectiva y necesita de una adecuada valoración de la arteria pudenda interna contralateral para no comprometer el flujo arterial del pene. ${ }^{12}$ Las principales complicaciones derivadas de la embolización de la arteria pudenda incluyen gangrena del pene, isquemia glútea, absceso perineal, migración del material de embolización y disfunción eréctil.

Los materiales utilizados para la embolización pueden ser reabsorbibles (coágulos autólogos o gelatinas absorbibles) o permanentes (microcoils, N-butil-cianocrilato o acetato de polivinilo). La ventaja de los materiales reabsorbibles es que la obstrucción al flujo sanguíneo es temporal, lo que permite recuperar la función eréctil cuando el material desaparece, pero presentan mayor riesgo de recidiva si el material se reabsorbe precozmente. ${ }^{13}$

En adultos, la embolización con sustancias no reabsorbibles consigue la resolución del priapismo en el $78 \%$ de los casos, con una incidencia de disfunción eréctil del 39\%, mientras que, en los casos en que se utilizan sustancias reabsorbibles, el priapismo se resuelve en el $74 \%$ de los pacientes, y existe disfunción eréctil en el 5\%. ${ }^{14}$ En la literatura, 30 niños con priapismo arterial han sido tratados mediante embolización con coágulos autólogos y se resolvió el cuadro en 24. Las recidivas se trataron satisfactoriamente

FIGURA 3. Diagrama de actuación ante la presencia de priapismo infantil

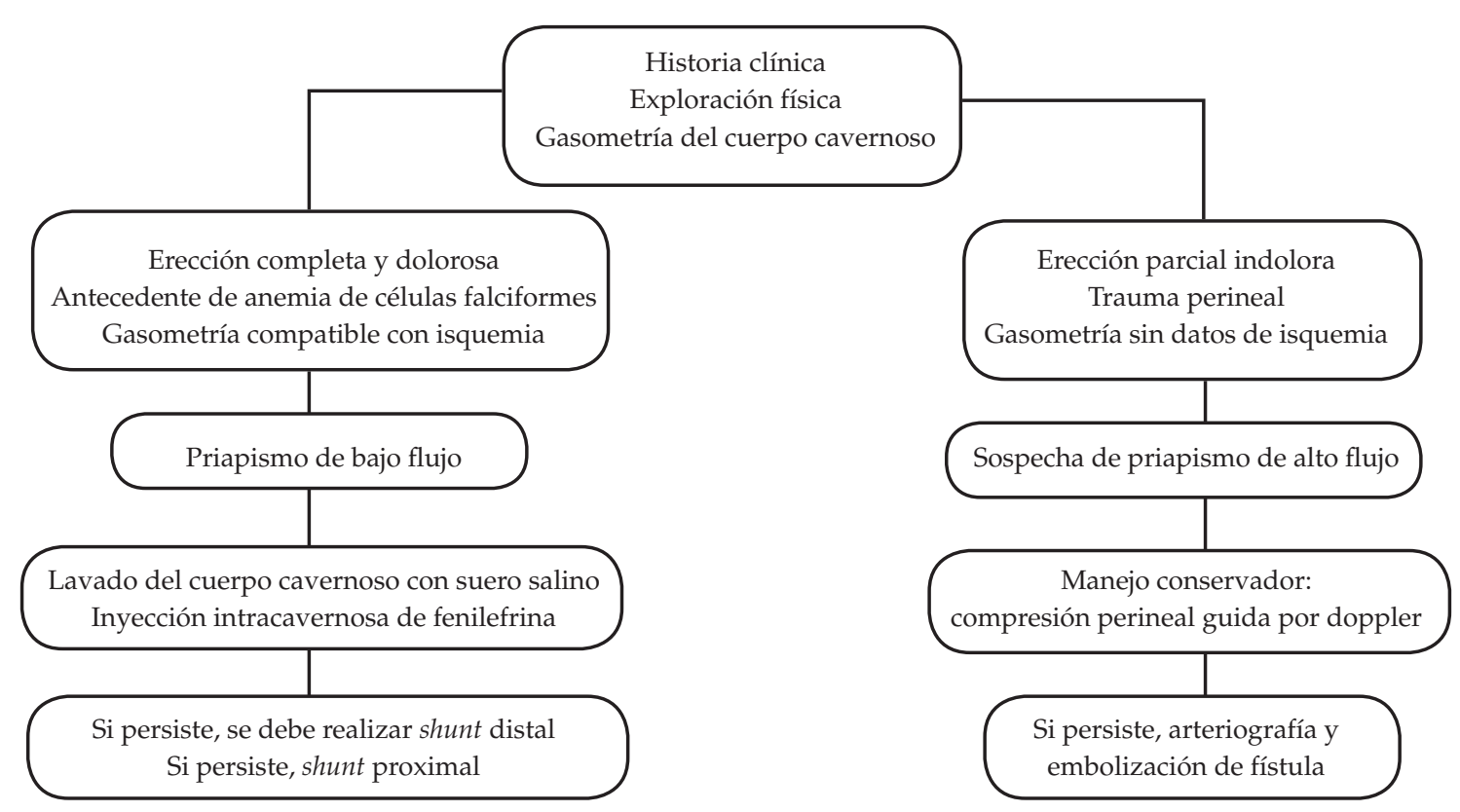


mediante embolización con microcoils, y no se encontró ningún caso de disfunción eréctil posterior. ${ }^{5}$

\section{CONCLUSIONES}

El priapismo de alto flujo es una rara complicación de los traumatismos perineales en pacientes pediátricos. El conocimiento de la fisiopatología de este trastorno es necesario para poder identificarlo y establecer el tratamiento definitivo, del que existen varias alternativas publicadas. La embolización de la fístula arteriocavernosa con sustancias reabsorbibles constituye una alternativa de tratamiento en esta patología con baja morbilidad. Se precisa un abordaje multidisciplinar que incluya pediatras, urólogos y radiólogos vasculares especializados.

\section{REFERENCIAS}

1. Broderick GA, Kadioglu A, Bivalacqua TJ, Ghanem H, etal. Priapism: pathogenesis, epidemiology, and management. J Sex Med 2010;7(Pt 2):476-500.

2. Eland IA, Van der Lei J, Stricker BH, Sturkenboom MJ. Incidence of priapism in the general population. Urology 2001;57(5):970-2.

3. Corbetta JP, Duran V, Burek C, Sager C, et al. High flow priapism: diagnosis and treatment in pediatric population. Pediatr Surg Int 2011;27(11):1217-21.

4. Rodríguez Villalba R, García S, Puigvert Martínez A, Pomerol Montseny J, Munárriz R. Priapismo. Actas Urol Esp 2005;29(10):961-8.
5. Mockford K, Weston M, Subramaniam R. Management of high-flow priapism in paediatric patients: a case report and review of the literature. J Pediatr Urol 2007;3(5):404-12.

6. Donalson J, Rees R, Steinbrecher H. Priapism in children: a comprehensive review and clinical guideline. J Pediatr Urol 2014;10(1):11-24.

7. Marotte JB, Brooks JD, Sze D, Kennedy IWA. 2nd. Juvenile posttraumatic high-flow priapism: current management dilemmas. J Pediatr Surg 2005;40(4):e25-8.

8. Rodríguez Corchero J, García Merino F, Cruz Navarro N, Barrero Candau R, et al. Priapismo arterial en paciente prepúber. Tratamiento conservador. Arch Esp Urol 2004;57(7):759-61.

9. Kumar R, Shrivastava DN, Seth A. Spontaneous resolution of delayed onset, posttraumatic high-flow priapism. J Postgtrad Med 2006;52(4):298-9.

10. Volgger H, Pfefferkorn S, Hobisch A. Posttraumatic highflow priapism in children: noninvasive treatment by color Doppler ultrasound-guided perineal compression. Urology 2007;70(3):590.e3-5.

11. Tonseth KA, Egge T, Kolbenstvedt A, Hedlund H. Evaluation of patients after treatment of arterial priapism with selective micro-embolisation. Scand J Urol Nephrol 2006;40(1):49-52.

12. Emir L, Tekgül S, Karabulut A, Oskay K, Erol D. Management of post-traumatic arterial priapism in children: presentation of a case and review of the literature. Int Urol Nephrol 2002;34(2):237-40.

13. Oztürk MH, Gümüş $M$, Dönmez H, Peynircioğlu B, et al. Materials in embolotherapy of high-flow priapism: results and long-term follow-up. Diagn Interv Radiol 2009;15(3):215-20.

14. Montague DK, Jarow J, Broderick GA, Dmochowski RR, et al. American Urological Association guideline on the management of priapism. J Urol 2003;170(4 Pt 1):1318-24. 